\title{
Microglial activation induced by LPS mediates excitation of neurons in the hypothalamic paraventricular nucleus projecting to the rostral ventrolateral medulla
}

\author{
Tae Hee Han, Heow Won Lee, Eun A Kang, Min Seok Song, So Yeong Lee * E Pan Dong Ryu* \\ Laboratory of Veterinary Pharmacology, College of Veterinary Medicine and Research Institute for Veterinary Science, Seoul National \\ University, Seoul 08826, Korea
}

\begin{abstract}
Microglia are known to be activated in the hypothalamic paraventricular nucleus (PVN) of rats with cardiovascular diseases. However, the exact role of microglial activation in the plasticity of presympathetic PVN neurons associated with the modulation of sympathetic outflow remains poorly investigated. In this study, we analyzed the direct link between microglial activation and spontaneous firing rate along with the underlying synaptic mechanisms in PVN neurons projecting to the rostral ventrolateral medulla (RVLM). Systemic injection of LPS induced microglial activation in the PVN, increased the frequency of spontaneous firing activity of PVN-RVLM neurons, reduced GABAergic inputs into these neurons, and increased plasma NE levels and heart rate. Systemic minocycline injection blocked all the observed LPS-induced effects. Our results indicate that LPS increases the firing rate and decreases GABAergic transmission in PVN-RVLM neurons associated with sympathetic outflow and the alteration is largely attributed to the activation of microglia. Our findings provide some insights into the role of microglial activation in regulating the activity of PVN-RVLM neurons associated with modulation of sympathetic outflow in cardiovascular diseases. [BMB Reports 2021; 54(12): 620-625]
\end{abstract}

\section{INTRODUCTION}

Activation of microglia in the brain is associated with neuronal plasticity in the central nervous system (1-4). Activated microglia increase spontaneous epileptiform discharges in the stratum pyramidale in the CA1 region of the hippocampus (4). Molecules

*Corresponding authors. So Yeong Lee, Tel: +82-2-880-1283; Fax: +822-879-0378; E-mail: leeso@snu.ac.kr; Pan Dong Ryu, Tel: +82-2-8801254; Fax: +82-2-879-0378; E-mail: pdryu@snu.ac.kr

https://doi.org/10.5483/BMBRep.2021.54.12.105

Received 2 August 2021, Revised 23 August 2021, Accepted 22 October 2021

Keywords: GABAergic transmission, Microglial activation, Neuronal activity, PVN-RVLM, Sympathetic outflow released by microglia potentiate N-Methyl-D-aspartic acid or $\mathrm{N}$-Methyl-D-aspartate receptor-mediated synaptic responses in organotypic cortical slice cultures (3). Microglia are activated in the hypothalamic paraventricular nucleus (PVN) of rats with myocardial infarction (MI)-induced cardiac damage or neurogenic hypertension (5-7). Sympathetic activity, which is regulated by presympathetic neurons in the hypothalamic PVN (8), and sympathetic activity-related neuronal firing rate are dramatically elevated in pathological conditions such as congestive heart failure (HF), following Ml and hypertension (9-12). Thus, many studies have suggested that elevated microglial activation in the brain may play a pivotal role in sympathetic hyperactivity seen in several pathological states including HF and hypertension $(5,6,13)$. However, despite accumulating evidence, the precise role of microglial activation in neuronal plasticity of presympathetic PVN neurons associated with the modulation of sympathetic outflow remains poorly investigated.

Presympathetic PVN neurons project to the rostral ventrolateral medulla (RVLM; PVN-RVLM neurons) or to the intermediolateral cell column of the spinal cord (IML; PVN-IML neurons) (14). The RVLM region possesses neurons playing a essential role in determining peripheral sympathetic vasomotor tone and blood pressure (BP), and thus RVLM neurons are described to as a cardiovascular center $(15,16)$. PVN-RVLM neurons play a critical role in the long-term control of the cardiovascular system in hypertension and HF $(10,12)$.

In this study, we investigated the direct link between microglial activation and the spontaneous firing rate along with the synaptic mechanisms in PVN neurons projecting to the RVLM.

\section{RESULTS}

LPS-induced microglial activation and its blockade by minocycline

As shown in Fig. 1C, systemic LPS injection induced activation of microglia as demonstrated by elevation in microglial specific protein Iba-1 expression, characterized by intensive staining and hypertrophy with large soma and thick processes in the PVN (Fig. 1C and insets) and a significantly higher number of Iba-1 immunoreactive pixels than the other groups (Fig. 1E, $P$

ISSN: 1976-670X (electronic edition)

Copyright (c) 2021 by the The Korean Society for Biochemistry and Molecular Biology

(c) This is an open-access article distributed under the terms of the Creative Commons Attribution Non-Commercial License (http://creativecommons.org/licenses/by-nc/4.0) which permits unrestricted non-commercial use, distribution, and reproduction in any medium, provided the original work is properly cited. 
$<0.001)$. However, pre-treatment with minocycline before LPS administration did affect LPS-induced morphological changes after LPS injection (Fig. 1D and insets), as shown in saline- and minocycline-treated rats (Fig. 1A, B, respectively). Collectively, the results indicate the activation of microglia by systemic LPS treatment. The LPS-induced microglial activation in the hypothalamic PVN was abrogated by systemic minocycline administration.

\section{LPS-induced increase of firing rate and its blockade by minocycline}

This study analyzed a total of 206 PVN-RVLM neurons (19 rats) using the brain slice patch-clamp technique. Supplementary Table 1 demonstrates the basic membrane properties of the four groups of PVN-RVLM neurons. None of these properties varied significantly between groups $(P>0.05)$. The majority of the labeled PVN-RVLM neurons recorded displayed spontaneous activity in saline (37 of 56 neurons, 66\%), minocycline (33 of 49 neurons, $67.3 \%$ ), LPS (35 of 47 neurons, $74.5 \%$ ), and minocycline + LPS group (37 of 54 neurons, 68.5\%) (Fig. $2 \mathrm{~A})$. The proportion of spontaneously active and silent neurons did not differ significantly between groups $(P>0.05$, using Fisher's exact test). The mean firing rate of LPS group (3.08 \pm $0.38 \mathrm{~Hz})$ was significantly higher than that of saline $(1.45 \pm$ $0.21 \mathrm{~Hz})$, minocycline $(1.74 \pm 0.30 \mathrm{~Hz})$, and minocycline + LPS groups $(1.70 \pm 0.24 \mathrm{~Hz})(\mathrm{P}<0.01$; Fig. $2 \mathrm{~B}$, left). In addition, the CV of the firing rate of LPS group $(0.52 \pm 0.07)$ was significantly lower than that of saline $(1.07 \pm 0.17)$, minocycline $(1.00 \pm 0.14)$, and minocycline + LPS groups $(0.99 \pm$ $0.14)$ ( $P<0.01$; Fig. 2B, right). As demonstrated in Fig. 2C, retrogradely labeled PVN-RVLM neurons were observed in the dorsomedial cap and the ventral regions and in the posterior regions of PVN. The inset of Fig. 2A shows a typical example
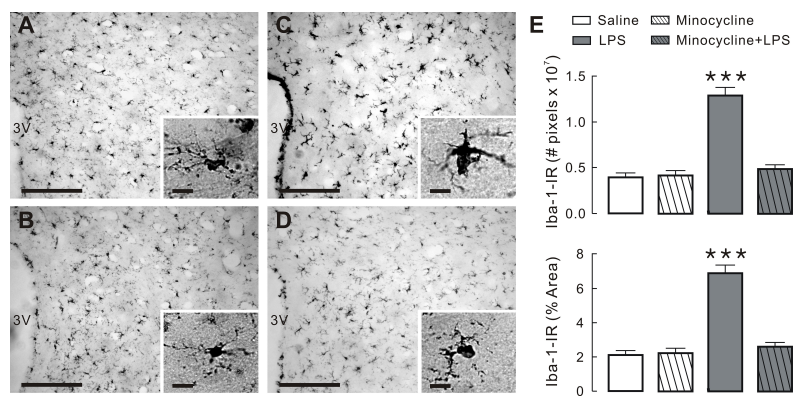

Fig. 1. Minocycline inhibits LPS-induced activation of microglia in PVN. (A-D) Images of PVN tissue slices stained with anti-lba-1. Images acquired from saline (A), minocycline (B), LPS (C) and minocycline + LPS groups (D), respectively. Note that insets in each figure show details of microglial morphology under high magnification. Scale bars are $200 \mu \mathrm{m}$ and $10 \mu \mathrm{m}$ (insets), respectively. (E) Quantification of lba-1 immunostaining in the PVN of four groups in $24 \mathrm{~h}$ following last challenge. $* * * \mathrm{P}<0.001$ by one-way ANOVA, followed by Newman-Keul's multiple comparison test. $\mathrm{N}$ $=3$ rats per group. of the injection site in the area of RVLM. Collectively, these results indicate that the LPS-induced increase in the firing rate was dependent on microglial activation in PVN-RVLM neurons.

\section{LPS-induced reduction of spontaneous IPSC frequency and its blockade by minocycline}

We analyzed the spontaneous miniature synaptic currents at the resting membrane potential $(-55$ to $-70 \mathrm{mV})$ in the whole cell voltage-clamp mode to record both outward and inward synaptic currents simultaneously (Fig. 3Aa). The outward currents were completely blocked by the $\mathrm{GABA}_{\mathrm{A}}$ receptor antagonist (20 $\mu \mathrm{M}$ bicuculline, BIC; Fig. 3Ab) indicating that these currents were IPSCs, whereas the inward currents were absolutely blocked by the antagonist cocktail of ionotropic glutamate receptors [20 $\mu \mathrm{M}$ 6-cyano-7-nitroquinoxaline-2,3-dione (CNQX) and $50 \mu \mathrm{M}$ dl-2-amino-5-phosphonopentanoic acid (AP5); Fig. 3Ac] confirming that these currents were EPSCs. The current records demonstrated in Fig. 3B demonstrate less frequent sIPSCs (upward events) in the PVN-RVLM neurons in LPS (Fig. 3Bc) than in saline (Fig. 3Ba), minocycline (Fig. 3Bb), and
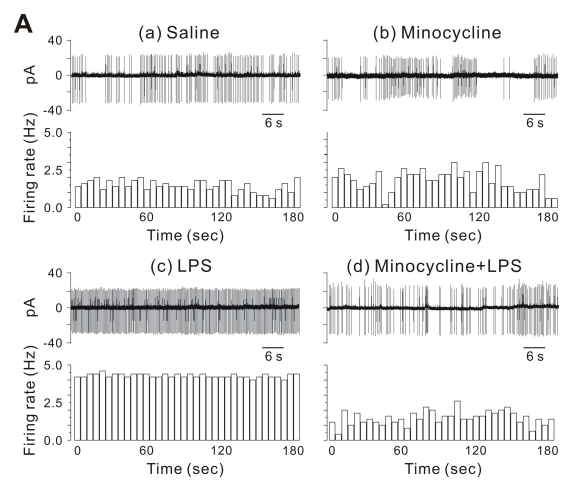

(d) Minocycline+LPS
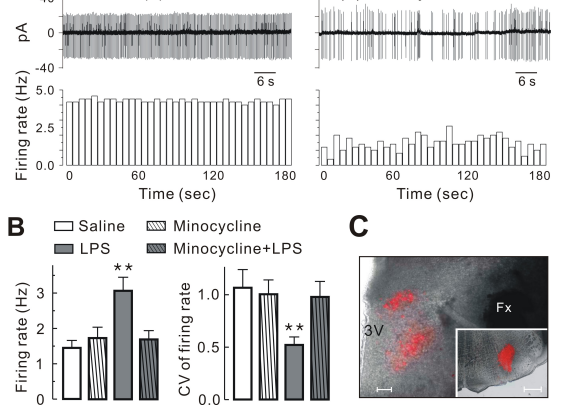

Minocycline

C
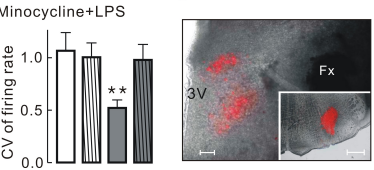

Fig. 2. Minocycline inhibits LPS-induced increase in firing rate of PVN-RVLM neurons. (A) Representative traces (upper panels) and time course histograms (lower panels) of spontaneous action potentials involving PVN-RVLM neurons in saline (a), minocycline (b), LPS (c), and minocycline + LPS group (d) under cell-attached voltage clamp mode (bin size $=5 \mathrm{~s}$ ). (B) Summary bar graphs showing the mean firing rate and CV of PVN-RVLM neurons $(n=37$ for saline; $n=33$ for minocycline; $n=35$ for LPS; and $n=37$ for minocycline + LPS). (C) Fluorescence images of the hypothalamic PVN regions showing distribution of retrograde-labeled PVN-RVLM neurons. Fluorescent images were superimposed onto images of the same slices obtained under bright light. The insets in $(\mathrm{C})$ represent the injection sites of fluorescent dye located in the RVLM of the medulla. Bars represent the mean \pm SEM. $* * P<0.01$ by one-way ANOVA followed by the Newman-Keul's multiple comparison test. 3V, third ventricle; Fx, fornix; Scale bars are $100 \mu \mathrm{m}$ (C) and $1 \mathrm{~mm}$ (insets in C). 
A
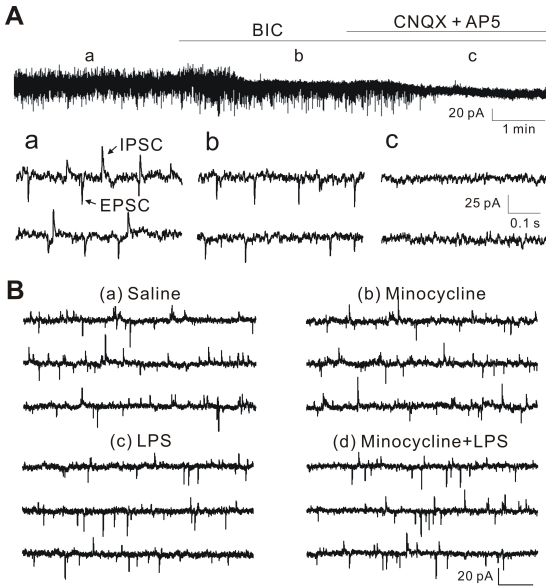

C
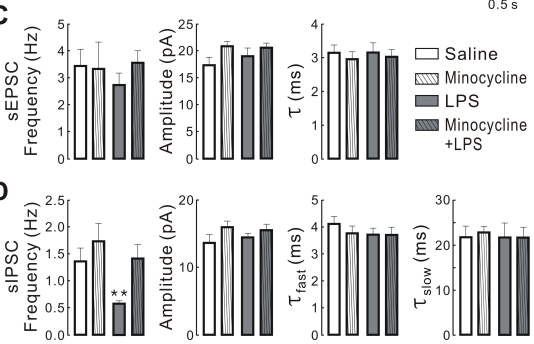

Fig. 3. Minocycline inhibits LPS-induced decrease in the frequency of spontaneous inhibitory postsynaptic currents (sIPSCs) in PVN-RVLM neurons. Spontaneous excitatory postsynaptic currents (EPSCs) and IPSCs recorded from PVN-RVLM neurons. (A) top: blockade of upward current by antagonists of $\mathrm{GABA}_{\mathrm{A}}$ receptor $(20 \mu \mathrm{M} \mathrm{Bic}$; b) and downward current by ionotropic glutamate receptor antagonist (50 $\mu \mathrm{M}$ AP5 and $20 \mu \mathrm{M}$ CNQX; c). Bottom: spontaneous EPSCs (downward) and IPSCs (upward) at expanded time scale before (a), during treatment with $\mathrm{BIC}(\mathrm{b})$, and $\mathrm{BIC}$ and $\mathrm{CNQX}+$ AP5 (c) at a holding potential of $-62 \mathrm{mV}$. (B) Representative traces of spontaneous synaptic currents recorded in the PVN-RVLM neurons derived from saline (a), minocycline (b), LPS (c), and minocycline + LPS groups (d). The synaptic currents were recorded in normal ACSF and at resting membrane potential. All the traces are continuous within each set of records. (C and D) summary bar graphs showing the mean frequency, amplitude, and decay time constants of sIPSCs and sEPSCs in PVN-RVLM neurons obtained from saline $(n=14)$, minocycline $(n=13)$, LPS $(n=$ $15)$, and minocycline + LPS group $(n=15)$, respectively. Values are mean SEM. $* * P<0.01$ by one-way ANOVA, followed by the Newman-Keul's multiple comparison test. $\tau_{\text {fast }}$ and $\tau_{\text {slow }}$ represent fast and slow decay time constants, respectively.

minocycline + LPS groups (Fig. 3Bd). The mean frequency of sIPSCs was significantly $(P<0.01)$ less in LPS $(0.59 \pm 0.05 \mathrm{~Hz})$ than in saline $(1.36 \pm 0.25 \mathrm{~Hz})$, minocycline $(1.72 \pm 0.35 \mathrm{~Hz})$, and minocycline + LPS group $(1.41 \pm 0.27 \mathrm{~Hz})$ (Fig. 3D). However, no significant differences in the mean amplitude of sIPSCs (saline vs. LPS vs. minocycline vs. minocycline + LPS, $13.60 \pm 1.25$ pA vs. $14.39 \pm 0.66$ pA vs. $15.90 \pm 0.94$ pA vs. $15.44 \pm 0.94 \mathrm{pA}, \mathrm{P}>0.05$; Fig. 3D) or in the two decay time constants of sIPSCs were observed between the four groups

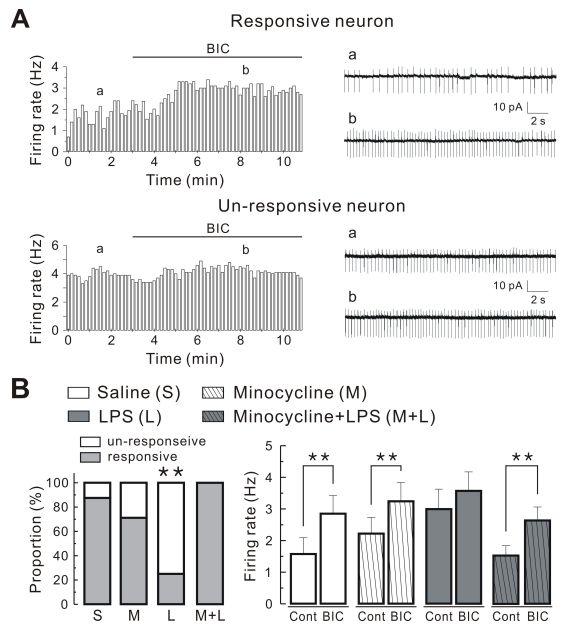

Fig. 4. Effect of Bic on firing activity of PVN-RVLM neurons in each group. (A) typical time course histograms (left panels) and representative traces (right panels) of firing activity of PVN-RVLM neurons before and after treatment with $20 \mu \mathrm{M}$ Bic (bin size = 10 s). Note two types of neurons responsive (upper panels) and un-responsive (lower panels) to Bic. (B) Left panel: comparison of the proportions of neurons un-responsive and responsive to bicuculline in PVN-RVLM neurons derived from saline, minocycline, LPS, and minocycline + LPS group. Right panel: summary bar graphs showing the effect of bicuculline on the firing rate of PVN-RVLM neurons obtained from saline $(n=8)$, minocycline $(n=7)$, LPS $(\mathrm{n}=8)$, and minocycline + LPS groups $(\mathrm{n}=8)$. Values are mean SEM. ${ }^{* * P}<0.01$ based on Student's t-test for paired samples or Fisher's exact test.

(saline vs. LPS vs. minocycline vs. minocycline + LPS: $\tau$ fast, $4.11 \pm 0.28 \mathrm{~ms}$ vs. $3.71 \pm 0.25 \mathrm{~ms}$ vs. $3.76 \pm 0.28 \mathrm{~ms}$ vs. $3.70 \pm 0.28 \mathrm{~ms}$, $\tau$ slow, $21.80 \pm 2.43 \mathrm{~ms}$ vs. $21.73 \pm 3.17 \mathrm{~ms}$ vs. $22.88 \pm 1.29 \mathrm{~ms}$ vs. $21.70 \pm 2.27 \mathrm{~ms} ; \mathrm{P}>0.05$; Fig. 3D). Further, in contrast to the changes in sIPSCs, no significant differences in frequency were detected between groups (saline vs. LPS vs. minocycline vs. minocycline + LPS, $3.46 \pm 0.61$ $\mathrm{Hz}$ vs. $2.75 \pm 0.44 \mathrm{~Hz}$ vs. $3.33 \pm 1.01 \mathrm{pA}$ vs. $3.54 \pm 0.48 \mathrm{pA}$, $P>0.05$; Fig. 3C), amplitude (saline vs. LPS vs. minocycline vs. minocycline + LPS, $17.44 \pm 1.48 \mathrm{pA}$ vs. $19.10 \pm 1.54 \mathrm{pA}$ vs. $20.89 \pm 1.01$ pA vs. $20.63 \pm 0.95$ pA, P > 0.05; Fig. 3C), and decay time constants (saline vs. LPS vs. minocycline vs. minocycline + LPS, $3.14 \pm 0.23 \mathrm{~ms}$ vs. $3.15 \pm 0.29 \mathrm{~ms}$ vs. $2.95 \pm 0.23 \mathrm{~ms}$ vs. $3.03 \pm 0.21 \mathrm{~ms}$; $\mathrm{P}>0.05$; Fig. $3 \mathrm{C})$ of sEPSCs (downward events) of PVN-RVLM neurons in each group. These results indicate that the LPS-induced activation of microglia preferentially alters GABA release into PVN-RVLM neurons.

\section{Blunted effect of bicuculline on the firing rate in the LPS group and its blockade by minocycline}

We analyzed the effect of endogenous GABA on PVN-RVLM neuronal activity in each group. Fig. 4 shows the response of some neurons to the $\mathrm{GABA}_{\mathrm{A}}$ receptor antagonist $(20 \mu \mathrm{M} \mathrm{BIC})$ 
(Fig. 4Aa) and the lack of response of others to bicuculline (Fig. 4Ab). Bath administration of bicuculline increased the firing rate of PVN-RVLM neurons tested in saline (7 of 8 neurons, $88 \%$ ), minocycline (5 of 7 neurons, $71 \%$ ), and minocycline + LPS groups (7 of 7 neurons, $100 \%$ ). The summarized data show that bicuculline significantly increased the firing rate (saline, from $1.58 \pm 0.51$ to $2.86 \pm 0.56 \mathrm{~Hz}$; minocycline, from $2.23 \pm 0.51$ to $3.25 \pm 0.59 \mathrm{~Hz}$; and minocycline + LPS, from $1.53 \pm 0.31$ to $2.63 \pm 0.43 \mathrm{~Hz} ; \mathrm{P}<0.01$, respectively; Fig. $4 \mathrm{~B}$, right). However, in the LPS group, bicuculline increased the firing rate in 2 of 8 neurons tested $(25 \%)$ and the changes were not significant (from $3.00 \pm 0.63$ to $3.58 \pm 0.59 ; \mathrm{P}>$ 0.05; Fig. $4 \mathrm{~B}$, right). In addition, we compared the proportion of neurons with altered firing rate following bicuculline treatment in each group. The proportion of responsive and un-responsive neurons differed significantly in PVN-RVLM neurons between each group ( $P<0.01$ using Fisher's exact test; Fig. $4 \mathrm{~B}$, left). Taken together, the results show that the effect of $\mathrm{GABA}_{\mathrm{A}}$ receptor blockade on the firing activity was blunted in PVN-RVLM neurons of the LPS group, reinforcing earlier observations that the LPS-induced activation of microglia reduces the release of synaptic GABA into PVN-RVLM neurons.

\section{LPS-induced sympathetic overactivation and its blockade by minocycline}

Plasma NE levels and heart rate can be regarded as markers of sympathetic tone $(17,18)$. Using the markers, we investigated whether LPS injection induced an increase in sympathetic activity and whether minocycline inhibited the LPS-induced effect. As shown in Supplementary Fig. 1A, the mean concentration of NE in the LPS group $(1.81 \pm 0.54 \mathrm{ng} / \mathrm{ml})$ was significantly higher than that of saline $(0.51 \pm 0.11 \mathrm{ng} / \mathrm{ml})$, minocycline $(0.63 \pm 0.11 \mathrm{ng} / \mathrm{ml})$, and minocycline + LPS groups $(0.72 \pm$ $0.23 \mathrm{ng} / \mathrm{ml})(\mathrm{P}<0.01$; Supplementary Fig. 1A). Similarly, the mean heart rate of LPS group (378 $\pm 26.7 \mathrm{bpm})$ was significantly higher than that of saline $(311 \pm 7.4 \mathrm{bpm})$, minocycline $(320 \pm 9.6 \mathrm{bpm})$, and minocycline + LPS groups $(328 \pm 9.2$ bpm) $(\mathrm{P}<0.01$; Supplementary Fig. 1B). Collectively, these results show that elevation in sympathetic tone depends on the activation of microglia.

\section{DISCUSSION}

Over the past decade, studies reported that the activation of microglia was closely related to sympathetic excitation such as HF and hypertension (19-21). However, the exact role and underlying mechanisms of microglial activation in such pathological conditions are still disputed (22). In the present study, we demonstrate that microglial activation in the PVN increases the spontaneous firing activity of PVN-RVLM neurons associated with sympathetic outflow and that the increase is largely attributed to a reduction in basal GABAergic inputs. This conclusion is supported by the systemic LPS-induced microglial activation in the PVN, increased frequency of spontaneous firing activity of PVN-RVLM neurons, reduced GABAergic inputs into PVN-RVLM neurons, and increased plasma NE levels and heart rate. Systemic minocycline injection inhibited all the observed LPS-induced effects. This study provides evidence suggesting that microglial activation in the PVN enhances neuronal excitation of PVN-RVLM neurons, which may in turn, lead to sympathetic overactivity commonly seen in pathological conditions including $\mathrm{HF}$ and hypertension.

Our results show the increased spontaneous firing activity of PVN-RVLM neurons via disinhibition of GABAergic inhibitory synaptic inputs. An important question raised by the present work relates to the role of activated microglia following systemic LPS injection in reducing GABAergic input into PVNRVLM neurons. Systemic LPS injection increases pro-inflammatory cytokines (PICs) such as interleukin (IL)-1, IL-6 and tumor necrosis factor- $\alpha$ (TNF- $\alpha$ ) in the PVN (23), with activated microglia constituting a major source of increase in PICs $(24,25)$. Especially, IL-1 $\beta$ increased the activity of electrophysiologically identified preautonomic parvocellular PVN neurons and decreased inhibiting GABAergic input via a prostaglandin E2-dependent mechanism, while glutamatergic input was not affected by IL-1 $\beta$ (26). Thus, it is possible that LPS-induced activation of microglia in the PVN releases IL-1 $\beta$, and thereby decreases GABA release, which in turn, leads to neuronal excitation of PVN-RVLM neurons. Another possibility is neurotoxic reactive astrocytes, which are induced by activated microglia via secretion of IL-1 $\alpha$, TNF and C1q complement component (27). Chen et al. showed that astrocytes were activated in the PVN of rats experiencing acute myocardial infarction (AMI), and astrocyte suppression by fluorocitrate, an inhibitor of astrocytic activation, reduced the expression of proinflammatory cytokines, neural activation, and sympathetic neural activity (28). Accordingly, aberrant astrocytes may play an active role in sympathetic excitation associated with the development and progression of hypertension and heart failure by releasing gliotransmitters and PICs (28-31). However, the function of cytokine receptors or reactive astrocytes on GABAergic and PVN-RVLM neurons has yet to be demonstrated. The precise roles of PICs and astrocytes in regulating the activity of PVN-RVLM neurons requires further study.

Minocycline, which permeates through the blood-brain barrier (32), is a semi-synthetic tetracycline antibiotic with anti-inflammatory activity in the central nervous system (33). Minocycline blocks microglial activation in the brain, without directly affecting astrocytes and neurons (34). Although the mechanism of minocycline action remains to be determined, our immunohistochemistry results (Fig. 1) demonstrate that minocycline treatment for three consecutive days effectively blocked the morphological changes associated with microglial activation in the PVN. Further, pre-treatment with minocycline before systemic LPS injection effectively abrogated all LPS-induced effects on PVN-RVLM neurons and markers of sympathetic activity (heart rate and plasma NE), while minocycline alone had no effect (see Figs. 2-4 and Supplementary Fig. 1). Previous reports 
indicated that central (intracerebroventricular injection; 50 $\mu \mathrm{g} / 2 \mu \mathrm{l} / \mathrm{L} \mathrm{min}$ ) and peripheral injection (intravenous injection; $100-150 \mathrm{mg} / \mathrm{kg}$ ) of minocycline suppresses motor activity in rats (35). However, the dose $(50 \mathrm{mg} / \mathrm{kg}$, for $3 \mathrm{~d}$ ) of minocycline used in this study attenuated LPS-induced neuroinflammation without affecting social behavior (36). These results indicate the limited role of minocycline in microglial inhibition under our experimental conditions.

In conclusion, our results provide some insights into the role of microglial activation in regulating neuronal activity in PVNRVLM neurons and further modulation of sympathetic outflow. Sustained exploration of the neurophysiological role of microglia in the PVN under pathological conditions accompanying sympathetic overactivity is warranted.

\section{MATERIALS AND METHODS}

Details on the used methods are provided in the expanded Materials and Methods section in the online data supplement.

\section{ACKNOWLEDGEMENTS}

This work was supported by National Research Foundation of Korea funded by the Ministry of Education, Science and Technology (2011-0025817).

\section{CONFLICTS OF INTEREST}

The authors have no conflicting interests.

\section{REFERENCES}

1. Ayoub AE and Salm AK (2003) Increased morphological diversity of microglia in the activated hypothalamic supraoptic nucleus. J Neurosci 23, 7759-7766

2. Liu B and Hong JS (2003) Role of microglia in inflammationmediated neurodegenerative diseases: mechanisms and strategies for therapeutic intervention. J Pharmacol Exp Ther $304,1-7$

3. Moriguchi S, Mizoguchi Y, Tomimatsu Y et al (2003) Potentiation of NMDA receptor-mediated synaptic responses by microglia. Brain Res Mol Brain Res 119, 160-169

4. Riazi K, Galic MA, Kuzmiski JB, Ho W, Sharkey KA and Pittman QJ (2008) Microglial activation and TNFalpha production mediate altered CNS excitability following peripheral inflammation. Proc Natl Acad Sci U S A 105, 17151-17156

5. Rana I, Stebbing M, Kompa A, Kelly DJ, Krum $\mathrm{H}$ and Badoer E (2010) Microglia activation in the hypothalamic PVN following myocardial infarction. Brain Res 1326, 96-104

6. Shi P, Diez-Freire C, Jun JY et al (2010) Brain microglial cytokines in neurogenic hypertension. Hypertension 56, 297-303

7. Dworak M, Stebbing M, Kompa AR, Rana I, Krum $\mathrm{H}$ and Badoer E (2012) Sustained activation of microglia in the hypothalamic PVN following myocardial infarction. Auton
Neurosci 169, 70-76

8. Swanson LW and Sawchenko PE (1980) Paraventricular nucleus: a site for the integration of neuroendocrine and autonomic mechanisms. Neuroendocrinology 31, 410-417

9. Anderson EA, Sinkey CA, Lawton WJ and Mark AL (1989) Elevated sympathetic nerve activity in borderline hypertensive humans. Evidence from direct intraneural recordings. Hypertension 14, 177-183

10. Dampney RA, Horiuchi J, Killinger S, Sheriff MJ, Tan PS and McDowall LM (2005) Long-term regulation of arterial blood pressure by hypothalamic nuclei: some critical questions. Clin Exp Pharmacol Physiol 32, 419-425

11. Li DP and Pan HL (2006) Plasticity of GABAergic control of hypothalamic presympathetic neurons in hypertension. Am J Physiol Heart Circ Physiol 290, H1110-H1119

12. Han TH, Lee K, Park JB et al (2010) Reduction in synaptic GABA release contributes to target-selective elevation of PVN neuronal activity in rats with myocardial infarction. Am J Physiol Regul Integr Comp Physiol 299, R129-R139

13. Badoer E (2010) Microglia: activation in acute and chronic inflammatory states and in response to cardiovascular dysfunction. Int J Biochem Cell Biol 42, 1580-1585

14. Pyner S and Coote JH (2000) Identification of branching paraventricular neurons of the hypothalamus that project to the rostroventrolateral medulla and spinal cord. Neuroscience 100, 549-556

15. Malpas SC (2010) Sympathetic nervous system overactivity and its role in the development of cardiovascular disease. Physiol Rev 90, 513-557

16. Kumagai $H$, Oshima N, Matsuura $T$ et al (2012) Importance of rostral ventrolateral medulla neurons in determining efferent sympathetic nerve activity and blood pressure. Hypertens Res 35, 132-141

17. Goldstein DS, McCarty R, Polinsky RJ and Kopin IJ (1983) Relationship between plasma norepinephrine and sympathetic neural activity. Hypertension 5, 552-559

18. Grassi G, Vailati S, Bertinieri G et al (1998) Heart rate as marker of sympathetic activity. J Hypertens 16, 1635-1639

19. Shen XZ, Li Y, Li L et al (2015) Microglia participate in neurogenic regulation of hypertension. Hypertension 66, 309-316

20. Diaz HS, Toledo C, Andrade DC, Marcus NJ and Del Rio $R$ (2020) Neuroinflammation in heart failure: new insights for an old disease. J Physiol 598, 33-59

21. Hu L, Zhang S, Ooi K et al (2020) Microglia-derived NLRP3 activation mediates the pressor effect of prorenin in the rostral ventrolateral medulla of stress-induced hypertensive rats. Neurosci Bull 36, 475-492

22. Takesue K, Kishi T, Hirooka Y and Sunagawa K (2017) Activation of microglia within paraventricular nucleus of hypothalamus is NOT involved in maintenance of established hypertension. J Cardiol 69, 84-88

23. Benicky J, Sanchez-Lemus E, Honda M et al (2011) Angiotensin II AT1 receptor blockade ameliorates brain inflammation. Neuropsychopharmacology 36, 857-870

24. Masson GS, Nair AR, Dange RB, Silva-Soares PP, Michelini LC and Francis J (2015) Toll-like receptor 4 promotes autonomic dysfunction, inflammation and microglia activation in the hypothalamic paraventricular nucleus: role of endoplasmic reticulum stress. PLoS One 10, e0122850 
25. Park JC, Han SH and Mook-Jung I (2020) Peripheral inflammatory biomarkers in Alzheimer's disease: a brief review. BMB Rep 53, 10-19

26. Ferri CC and Ferguson AV (2005) Prostaglandin E2 mediates cellular effects of interleukin-1 beta on parvocellular neurones in the paraventricular nucleus of the hypothalamus. J Neuroendocrinol 17, 498-508

27. Liddelow SA, Guttenplan KA, Clarke LE et al (2017) Neurotoxic reactive astrocytes are induced by activated microglia. Nature 541, 481-487

28. Chen J, Yin D, He X et al (2020) Modulation of activated astrocytes in the hypothalamus paraventricular nucleus to prevent ventricular arrhythmia complicating acute myocardial infarction. Int J Cardiol 308, 33-41

29. Del Rio R, Quintanilla RA, Orellana JA and Retamal MA (2015) Neuron-Glia crosstalk in the autonomic nervous system and its possible role in the progression of metabolic syndrome: a new hypothesis. Front Physiol 6, 350

30. Marina N, Teschemacher AG, Kasparov S and Gourine AV (2016) Glia, sympathetic activity and cardiovascular disease. Exp Physiol 101, 565-576
31. Allen NJ and Eroglu C (2017) Cell biology of astrocytesynapse interactions. Neuron 96, 697-708

32. Tomas-Camardiel M, Rite I, Herrera AJ et al (2004) Minocycline reduces the lipopolysaccharide-induced inflammatory reaction, peroxynitrite-mediated nitration of proteins, disruption of the blood-brain barrier, and damage in the nigral dopaminergic system. Neurobiol Dis 16, 190-201

33. Amin AR, Attur MG, Thakker GD et al (1996) A novel mechanism of action of tetracyclines: effects on nitric oxide synthases. Proc Natl Acad Sci U S A 93, 14014-14019

34. Tikka TM and Koistinaho JE (2001) Minocycline provides neuroprotection against $\mathrm{N}$-methyl-D-aspartate neurotoxicity by inhibiting microglia. J Immunol 166, 7527-7533

35. Kofman O, van Embden S, Alpert C and Fuchs I (1993) Central and peripheral minocycline suppresses motor activity in rats. Pharmacol Biochem Behav 44, 397-402

36. Henry CJ, Huang Y, Wynne A et al (2008) Minocycline attenuates lipopolysaccharide (LPS)-induced neuroinflammation, sickness behavior, and anhedonia. J Neuroinflammation 5, 15 